\title{
Antiresonant Reflecting Optical Waveguides Polarization Beam Splitters
}

\author{
Yang-Tung Huang, Member, IEEE, Chiou-Hung Jang, Shih-Hsin Hsu, Member, IEEE, \\ and Jau-Jan Deng, Member, IEEE
}

\begin{abstract}
Three different configurations of polarization beam splitters based on antiresonant reflecting optical waveguide (ARROW) structures are proposed. The polarization splittings are attained by utilizing various characteristics of basic ARROW and/or ARROW-B. In comparison with conventional waveguides, ARROW structures utilizing antiresonant reflection as the guiding mechanism can have large core sizes compatible with singlemode fibers and, thus, provide better coupling. Design principles and characteristics of these ARROW polarization beam splitters are discussed in detail, and the simulation results show that all these devices have good polarization discrimination with crosstalk $<-20 \mathrm{~dB}$.
\end{abstract}

Index Terms-Beam splitting, integrated optics, optical planar waveguides, polarization, waveguide couplers.

\section{INTRODUCTION}

$\mathbf{P}$ OLARIZATION beam splitters are important integratedoptic devices in fiber sensors and coherent optical communication systems. Polarization splittings can be realized by either manipulating different beat lengths for two polarizations [1]-[4], by designing the waveguides optically identical for one polarization while nonidentical for the other [5]-[9], or by employing resonant tunneling that inserts a middle high-index layer as the tunneling layer allowing complete coupling for one polarization while preventing coupling for the other [10].

The first polarization beam splitter based on an antiresonant reflecting optical waveguide (ARROW) structure has been demonstrated in [11]. In comparison with conventional waveguides, ARROWs feature large core sizes compatible to those of fibers [12]. For dual ARROW configurations, remote coupling is feasible [13], [14], and a novel power divider and a hybrid coupler can be realized by simply varying the air-cladding layer thickness as well [15], [16]. Besides, an ARROW-B has been proposed for polarization-insensitive

Manuscript received January 26, 2006; revised May 21, 2006. This work was supported in part by the National Science Council of the Republic of China under Contract NSC86-2221-E-009-040.

Y.-T. Huang is with the Department of Electronics Engineering and Institute of Electronics, National Chiao Tung University, Hsinchu 30010, Taiwan, R.O.C.

C.-H. Jang was with the Department of Electronics Engineering and Institute of Electronics, National Chiao Tung University, Hsinchu 30010, Taiwan, R.O.C. He is now with Intel Corporation, Hillsboro, OR 97124 USA.

S.-H. Hsu was with the Department of Electronics Engineering and Institute of Electronics, National Chiao Tung University, Hsinchu 30010, Taiwan, R.O.C. He is now with the Research Center for Applied Sciences, Academia Sinica, Taipei 11529, Taiwan, R.O.C. (e-mail: shhsu.ee85g@nctu.edu.tw).

J.-J. Deng was with the Department of Electronics Engineering and Institute of Electronics, National Chiao Tung University, Hsinchu 30010, Taiwan, R.O.C. He is now with E-Pin Optical Industry Co., Taipei 112, Taiwan, R.O.C.

Digital Object Identifier 10.1109/JLT.2006.879503 waveguiding [17], and its structure is capable of sustaining the fundamental modes of both polarizations while higher order modes are effectively filtered out due to large propagation losses.

In this paper, based on the features of ARROW and ARROW-B structures, three different structures of polarization beam splitters are presented, and the finite-difference beam propagation method (FD-BPM) simulation is applied to verify the designs. The first polarization beam splitter is based on basic ARROW and ARROW-B waveguides. The device structure and operating principles are presented, an example is given to demonstrate the design, and the crosstalk is calculated. Since the device length is a multiple of the coupling lengths, a modified structure whose coupling lengths are relatively short will be more attractive. It has been found that the degree of symmetry as well as the coupling length of a dual ARROW can be tuned by varying the air-cladding thickness [16]. Based on this, we propose a polarization beam splitter based on an adiabatically tapered ARROW structure. The eigenmode expansion method is applied to analyze and optimize this kind of structure with step discontinuities. Nonetheless, the device length could be dramatically shortened if the polarization splitting can be achieved during a single coupling process. This concept is utilized in the third polarization beam splitter, which is based on an asymmetric ARROW structure.

\section{Polarization Beam Splitter Based on Basic ARROW AND ARROW-B WAVEGUIDES}

It is known that one of the features of a conventional ARROW as shown in Fig. 1(a) is that only TE-polarized waves can propagate without significant losses since the reflectivities of TM-polarized waves from Fabry-Pérot cavities are relatively low [12]. However, an ARROW-B structure has been proposed for polarization-insensitive waveguiding [17]. In contrast to conventional ARROWs, the refractive index of the first cladding in an ARROW-B is lower than that of the core, as shown in Fig. 1(b). Since this layer is so thin that frustrated total internal reflections occur, the evanescent field can reach the second cladding layer, which satisfies the transverse antiresonance condition (discussed below). Because the total internal reflection dominates the overall reflection, the reflectivity in an ARROW-B is less polarization dependent than that in a conventional ARROW. The structure with losses lower than $0.5 \mathrm{~dB} / \mathrm{cm}$ for the fundamental modes $\left(\mathrm{TE}_{0}\right.$ and $\left.\mathrm{TM}_{0}\right)$ and higher than $10 \mathrm{~dB} / \mathrm{cm}$ for all other higher order modes has been demonstrated [17]. 


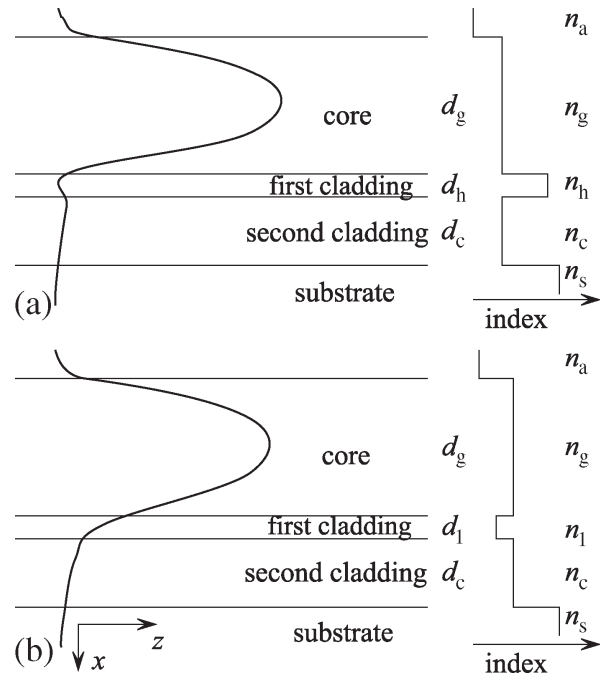

Fig. 1. (a) Conventional ARROW structure. (b) ARROW-B structure.

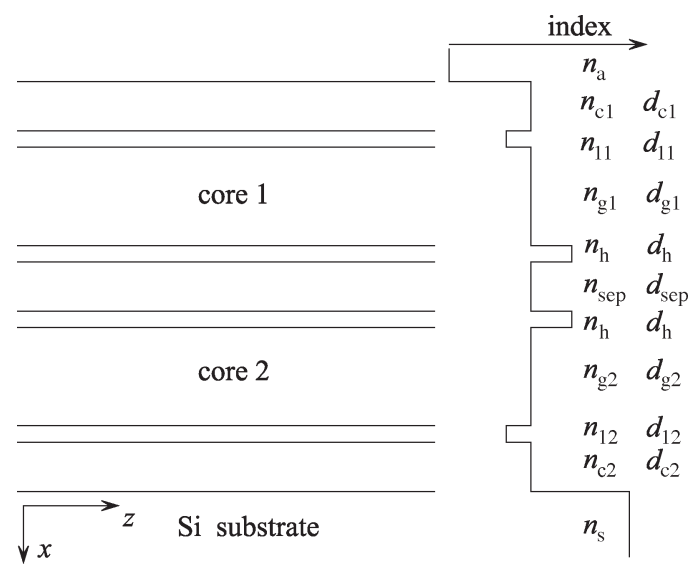

Fig. 2. Schematic view of the polarization beam splitter based on basic ARROW and ARROW-B waveguides.

The structure of the polarization beam splitter based on combining the mentioned features of conventional ARROW and ARROW-B waveguides is shown in Fig. 2. When an optical beam comprised of both polarizations is launched at core 1 , the fundamental even and odd modes for the both polarizations are equally excited, and the cumulated phase differences between the two fundamental modes for TE and TM waves are

$$
\begin{aligned}
k_{0} L \Delta N_{\mathrm{TE}} & \equiv k_{0} L\left(N_{\mathrm{TE}, e}-N_{\mathrm{TE}, o}\right)=m \pi \\
k_{0} L \Delta N_{\mathrm{TM}} & \equiv k_{0} L\left(N_{\mathrm{TM}, e}-N_{\mathrm{TM}, o}\right)=l \pi
\end{aligned}
$$

respectively. $k_{0}$ is the free-space wavenumber, $L$ is the device length, and $N$ is the real part of complex effective indices. The subscripts of $N$ denote the polarization and mode, respectively, e.g., $N_{\mathrm{TE}, e}$ denotes the real part of the effective index of the even mode for the TE polarization. To separate TE- and TMpolarized waves, $L$ is adjusted to make $m$ and $l$ in (1) and (2) one even integer while the other odd. Subtracting (1) from (2), we get

$$
k_{0} L\left(\Delta N_{\mathrm{TM}}-\Delta N_{\mathrm{TE}}\right)=(l-m) \pi=s \pi .
$$

We can solve $L$ from (3) by setting $s$ as an odd integer, and then substitute the derived $L$ back to (1) and (2) to check if $m$ and $l$ are both integers.

With the operating wavelength $\lambda=0.6328 \mu \mathrm{m}$, we exploit $\mathrm{Si}, \mathrm{SiO}_{2}, \mathrm{SiON}$, and NA45 glass as the material system to demonstrate the design. With $\mathrm{Si}(n=3.50)$ being the substrate, NA45 glass $(n=1.54)$ acts as the guiding layers (core 1 and core 2), the separation layer, and the cladding layers, and $\mathrm{SiO}_{2}$ $(n=1.46)$ acts as the low-index cladding layers. That is, $n_{s}=$ $3.50, n_{g_{1}}=n_{g_{2}}=n_{\text {sep }}=n_{c_{1}}=n_{c_{2}}=1.54$, and $n_{l_{1}}=n_{l_{2}}=$ 1.46. The corresponding thicknesses for core 1 and core 2 are chosen to be $4 \mu \mathrm{m}$ to have efficient coupling with fibers [15], [18] and $2 \mu \mathrm{m}$ for the cladding layers and the separation layer to attain the transverse antiresonance condition [12], i.e.,

$$
\begin{aligned}
& d_{j} \simeq \frac{\lambda}{4 n_{j}}\left[1-\left(\frac{n_{g_{1}}}{n_{j}}\right)^{2}+\left(\frac{\lambda}{2 n_{j} d_{g_{1}}}\right)^{2}\right]^{-(1 / 2)} \\
& \cdot\left(2 Q_{j}+1\right), \quad Q_{j}=0,1,2,3, \ldots
\end{aligned}
$$

where $j=h$, sep, $c_{1}$, and $c_{2} . Q_{j}$ is the order of antiresonant condition and can be chosen arbitrarily. The thicknesses of the low-index layers $d_{l_{1}}$ and $d_{l_{2}}$ are both chosen as $0.3 \mu \mathrm{m}$ such that only the fundamental even and odd modes for both polarizations can propagate without significant losses $(<0.5 \mathrm{~dB} / \mathrm{cm})$ while all other higher order modes are filtered out due to large propagation losses $(>10 \mathrm{~dB} / \mathrm{cm})$.

The crosstalk of the polarization beam splitter can be defined as

$$
\mathrm{CT}_{\mathrm{TE}}=10 \cdot \log _{10} \frac{P_{1}^{\mathrm{TE}}}{P_{2}^{\mathrm{TE}}}, \quad \mathrm{CT}_{\mathrm{TM}}=10 \cdot \log _{10} \frac{P_{2}^{\mathrm{TM}}}{P_{1}^{\mathrm{TM}}}
$$

where $P_{1}$ and $P_{2}$ are the output powers at core 1 and core 2, respectively. To have the crosstalk as small as possible, i.e., to have $m$ and $l$ exact integers, the choice of the high-index cladding layer $n_{h}$ is the major concern since $n_{h}$ is the dominant factor of $\left(\Delta N_{\mathrm{TM}}-\Delta N_{\mathrm{TE}}\right)$. With the SiON technology, which can vary the refractive index of $\mathrm{SiON}$ from $1.45\left(\mathrm{SiO}_{2}\right)$ to $1.98\left(\mathrm{Si}_{3} \mathrm{~N}_{4}\right)$ [7], we continuously vary $n_{h}$ from 1.58 to 1.98 with corresponding thicknesses and solve (1) and (3). A solution diagram can be constructed as shown in Fig. 3, whose right axis is the $m$ value in (1). It is shown that solutions with $m$ ranging from 2 to 7 are adequate choices. With the corresponding $n_{h}$, the polarization splitting with crosstalks for both polarizations approaching zero is attainable since $m$ and, thus, $l$ are taken as exact integers.

In real cases, the exact solutions of $n_{h}$ might be hard to realize, thus a reasonable value around the exact solution should be chosen. The coupling length can be determined as

$$
L_{C, p}=\frac{\lambda}{2\left|N_{p, e}-N_{p, o}\right|}
$$

where the subscript $p$ denotes the polarization, and the device length $L$ is adjusted between $m \cdot L_{C, \mathrm{TE}}$ and $l \cdot L_{C, \mathrm{TM}}$, i.e., $l \cdot L_{C, \mathrm{TM}} \leq L \leq m \cdot L_{C, \mathrm{TE}}$. Note that if $n_{h}$ can be controlled at the exact solution, then $l \cdot L_{C, \mathrm{TM}}=L=m \cdot L_{C, \mathrm{TE}}$, and thus, crosstalks for both polarizations are zero. 


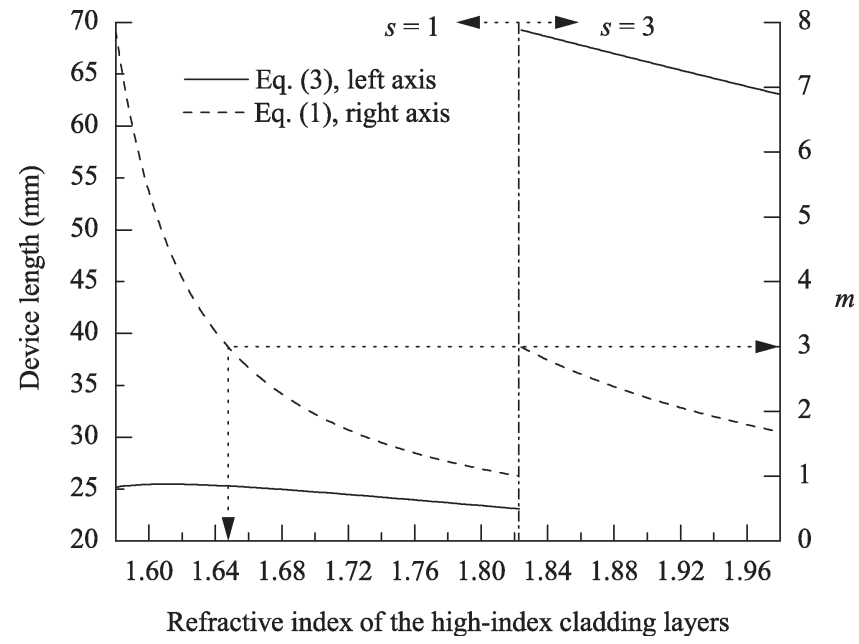

Fig. 3. Solution diagram for choosing $n_{h}$.

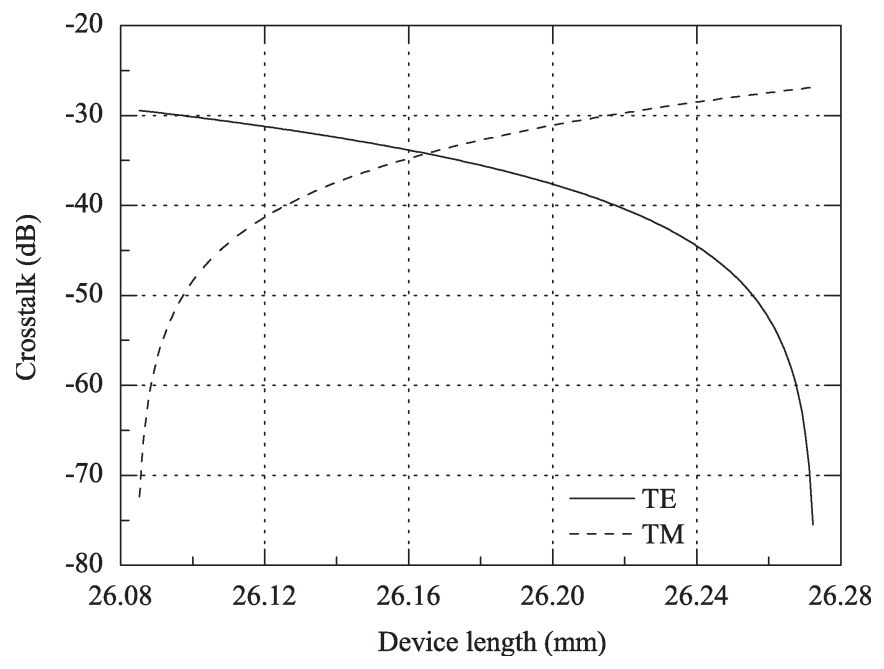

Fig. 4. Crosstalk versus device length for both polarizations.

As a design example, we choose the solution with $m=3$, and then $n_{h} \approx 1.648$. For a reasonable choice of $n_{h}$, we take $n_{h}=1.65$, and the corresponding thickness is $d_{h}=0.265 \mu \mathrm{m}$. It is found that $L_{C, \mathrm{TE}} \approx 8.76 \mathrm{~mm}$ and $L_{C, \mathrm{TM}} \approx 6.52 \mathrm{~mm}$. Choosing the device length $L$ as $4 L_{C, \mathrm{TM}} \leq L \leq 3 L_{C, \mathrm{TE}}$, the crosstalks for both polarizations can be kept below $-30 \mathrm{~dB}$ within a broad range $(\approx 120 \mu \mathrm{m})$, as depicted in Fig. 4.

The FD-BPM is applied to verify the design. In either polarization, a unit-power wave composed of the superposition of the even and odd modes is launched at core 1 . Based on Fig. 4, the device length is chosen as $26.16 \mathrm{~mm}$, and the power evolution profiles of TE- and TM-polarized waves are shown in Fig. 5(a) and (b), respectively. Since the propagation losses of the fundamental modes are lower than $0.07 \mathrm{~dB} / \mathrm{cm}$ for TEpolarized waves and around $0.3 \mathrm{~dB} / \mathrm{cm}$ for that of TM, the insertion loss of the device for the TM polarization $(\approx 0.75 \mathrm{~dB})$ is considerably higher than that for TE $(\approx 0.17 \mathrm{~dB})$.

\section{Polarization Beam Splitter Based on Adiabatically TAPERED ARROW}

As described in the preceding section, the device length is somewhat lengthy for integrated optics due to the relatively
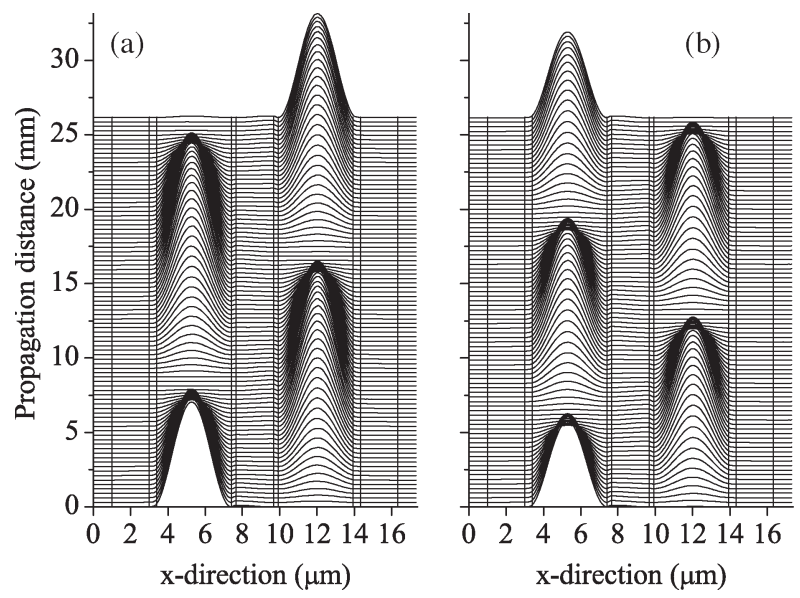

Fig. 5. Power evolution profiles. (a) TE polarization. (b) TM polarization.

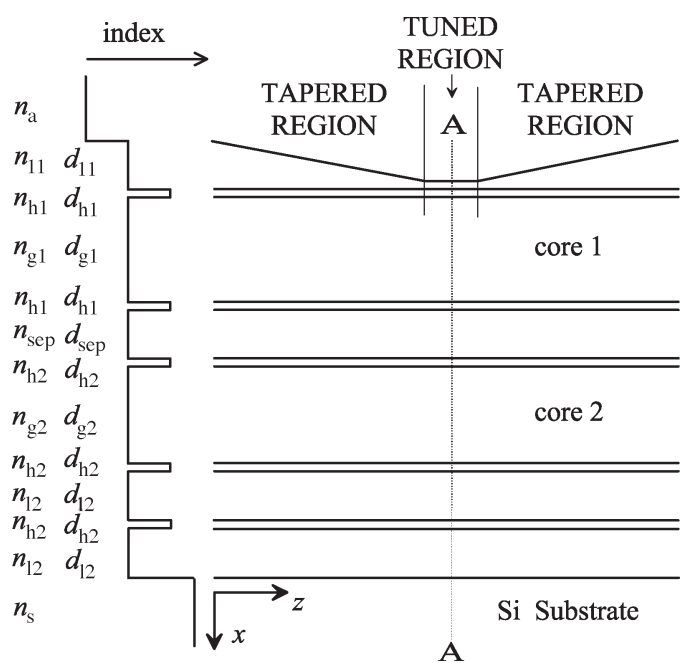

Fig. 6. Schematic view of the polarization beam splitter based on adiabatically tapered ARROW.

long coupling lengths, which correspond to the small difference between the effective indices of the fundamental even and odd modes. In this section, a novel type of polarization beam splitter with reduced device length is proposed. Based on our previous investigations, in dual ARROWs, the effective indices of fundamental even and odd modes for TE polarization are dependent on the air-cladding thickness [15], [16]. In fact, the same characteristics also occur on TM polarization. Thus, by tapering the air-cladding layer, the value of $\left(\Delta N_{\mathrm{TM}}-\Delta N_{\mathrm{TE}}\right)$ in (3) can be increased to have a shorter device length.

The configuration of the polarization beam splitter $\left(n_{a} / n_{l_{1}} / n_{h_{1}} / n_{g_{1}} / n_{h_{1}} / n_{\text {sep }} / n_{h_{2}} / n_{g_{2}} / n_{h_{2}} / n_{l_{2}} / n_{h_{2}} / n_{l_{2}} / n_{s}\right)$ is depicted in Fig. 6. The structure along the $z$-axis is symmetric about the A-A section. The air-cladding layer is adiabatically tapered from the input end, which possesses a high degree of symmetry, to the tuned region, whose length is to be adjusted, and then adiabatically tapered again to the output end. In contrast to original dual ARROWs [15], an additional Fabry-Pérot cavity is inserted in between core 2 and the substrate for the sake of sustaining the fundamental even and odd TM modes while still keeping higher order modes with large propagation losses. 
The tapering angle of the air-cladding layer must be made gradual enough to attain the adiabatic condition, i.e., making sure that no significant mode conversion occurs. This adiabatic condition is analyzed using the eigenmode expansion method by modeling the tapered structure as a series of staircases with small and abrupt discontinuities [16].

When an optical beam containing TE and TM polarizations is launched at core 1, assuming the perfect adiabatic condition, the cumulated phase differences of the fundamental even and odd modes for both polarizations are

$$
\begin{aligned}
\Phi_{1, \mathrm{TE}}+k_{0} L \Delta N_{\mathrm{TE}}+\Phi_{2, \mathrm{TE}} & =m \pi \\
\Phi_{1, \mathrm{TM}}+k_{0} L \Delta N_{\mathrm{TM}}+\Phi_{2, \mathrm{TM}} & =l \pi
\end{aligned}
$$

where $\Phi_{1, \mathrm{TE}}$ and $\Phi_{2, \mathrm{TE}}$ are the cumulated phase differences experienced in the first and second tapered regions for TE waves, respectively, and $\Phi_{1, \mathrm{TM}}$ and $\Phi_{2, \mathrm{TM}}$ are those for TM waves. Since the two tapered regions are identical, $\Phi_{1, \mathrm{TE}}=$ $\Phi_{2, \mathrm{TE}} \equiv \Phi_{\mathrm{TE}}$ and $\Phi_{1, \mathrm{TM}}=\Phi_{2, \mathrm{TM}} \equiv \Phi_{\mathrm{TM}} . L$ and $\Delta N$ denote the length and the effective index difference of the tuned region, respectively, and the others are defined as those in Section II. For acquiring the separation of TE and TM waves, $L$ must be adjusted to make $m$ and $l$ in (7) and (8) one even integer while the other odd. Subtracting (7) from (8), we have

$$
k_{0} L\left(\Delta N_{\mathrm{TM}}-\Delta N_{\mathrm{TE}}\right)+2\left(\Phi_{\mathrm{TM}}-\Phi_{\mathrm{TE}}\right)=(l-m) \pi=s \pi .
$$

We solve $L$ from (9) by setting $s$ as an odd integer, and then substitute the derived $L$ back to (7) and (8) to check if $m$ and $l$ are integers.

For optimizing the design, i.e., to make the device length as short as possible, we have to make $\left(\Delta N_{\mathrm{TM}}-\Delta N_{\mathrm{TE}}\right)$ as large as possible while still keeping $m$ and $l$ both integers. Since the adiabatic condition is dependent on the air-cladding layer thickness of the tuned region, after choosing a tapering angle and adjusting the air-cladding layer thickness to minimize the device length, we have to check if the adiabatic condition is still preserved.

Similar to the previous section, we exploit $\mathrm{Si}, \mathrm{SiO}_{2}$, and a glass mixture $\left(\mathrm{SiO}_{2}\right.$ and $\left.\mathrm{BaO}\right)$ as the material system of the design example. With $\mathrm{Si}$ being the substrate $\left(n_{s}=3.50\right)$, $\mathrm{SiO}_{2}$ acts as the guiding layers, the separation layer, and the low-index layers $\left(n_{g_{1}}=n_{g_{2}}=n_{\text {sep }}=n_{l_{1}}=n_{l_{2}}=1.46\right)$, and the glass mixture acts as the high-index layers $\left(n_{h_{1}}=n_{h_{2}}=\right.$ 1.50). Choosing $d_{g_{1}}=d_{g_{2}}=4 \mu \mathrm{m}$, the corresponding thickness of each layer is determined from (4), i.e., $d_{l_{2}}=d_{\text {sep }}=$ $2 \mu \mathrm{m}$ and $d_{h_{1}}=d_{h_{2}}=0.448 \mu \mathrm{m}$. To make the device possess a high degree of symmetry at the input end, the thickness of the air-cladding layer $d_{l_{1}}$ has to be adjusted. Fig. 7 shows the modal power ratio of the fundamental even and odd modes between two cores versus the air-cladding layer thickness for both polarizations. It is observed that the optimum thickness is $1.91 \mu \mathrm{m}$ for the TE polarization and $1.97 \mu \mathrm{m}$ for that of TM. Since throughout the device, the odd mode experiences a little larger propagation loss than the even mode, we can choose $d_{l_{1}}=1.91 \mu \mathrm{m}$ in the design example, and the deviation of the power symmetry for TM modes could be some-

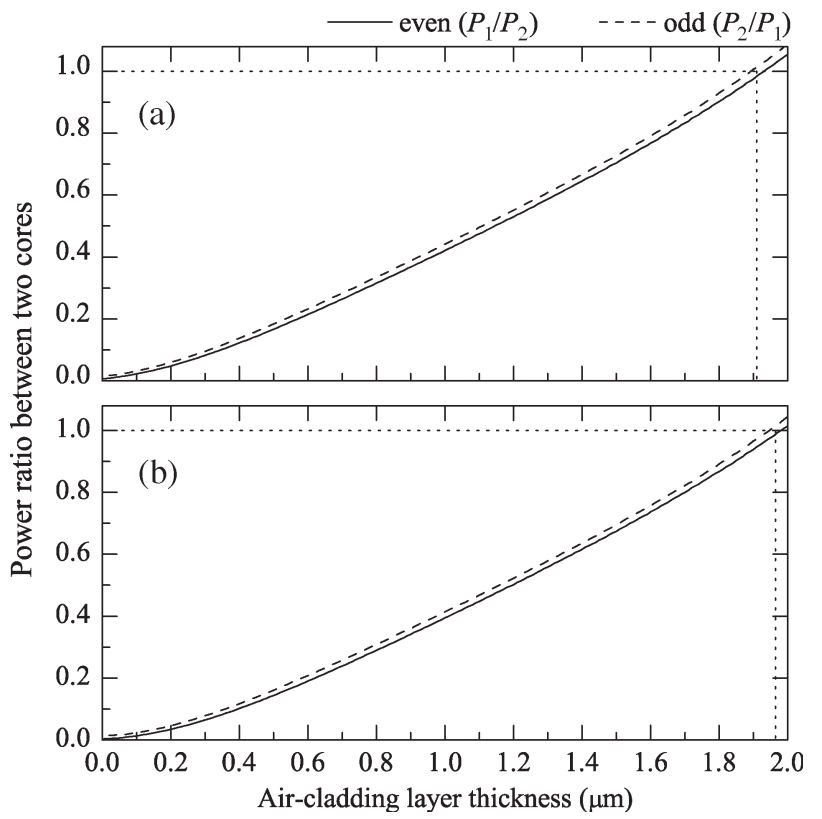

Fig. 7. Modal power ratios of the fundamental even and odd modes between two cores versus the air-cladding layer thickness $d_{l_{1}}$. (a) TE polarization. (b) TM polarization.

TABLE I

EXCITATION EFFICIENCIES OF THE FIRST TEN MODES FOR BOTH POLARIZATIONS AT THE INPUT END

\begin{tabular}{|c|c|c|c|}
\hline Modes & $\begin{array}{c}\text { Effective Index } \\
\text { (real part) }\end{array}$ & $\begin{array}{c}\text { Propagation } \\
\text { Loss }(\mathrm{dB} / \mathrm{cm})\end{array}$ & $\begin{array}{c}\text { Excitation } \\
\text { Efficiency (\%) }\end{array}$ \\
\hline \hline $\mathrm{TE}_{0}$ & 1.457918 & 0.031 & 39.76 \\
\hline $\mathrm{TE}_{1}$ & 1.457789 & 0.039 & 40.37 \\
\hline $\mathrm{TE}_{2}$ & 1.453922 & 23.296 & 0.13 \\
\hline $\mathrm{TE}_{3}$ & 1.453356 & 25.974 & 0.01 \\
\hline $\mathrm{TE}_{4}$ & 1.452315 & 28.847 & 1.08 \\
\hline $\mathrm{TE}_{5}$ & 1.450955 & 42.919 & 0.70 \\
\hline $\mathrm{TE}_{6}$ & 1.449498 & 49.918 & 0.01 \\
\hline $\mathrm{TE}_{7}$ & 1.448435 & 55.120 & 0.02 \\
\hline $\mathrm{TE}_{8}$ & 1.442544 & 8.285 & 0.03 \\
\hline $\mathrm{TE}_{9}$ & 1.440783 & 10.508 & 38.56 \\
\hline \hline $\mathrm{TM}_{0}$ & 1.457923 & 0.223 & 40.73 \\
\hline $\mathrm{TM}_{1}$ & 1.457780 & 0.276 & 0.24 \\
\hline $\mathrm{TM}_{2}$ & 1.453940 & 119.161 & 0.07 \\
\hline $\mathrm{TM}_{3}$ & 1.453371 & 170.663 & 1.20 \\
\hline $\mathrm{TM}_{4}$ & 1.452250 & 184.678 & 1.01 \\
\hline $\mathrm{TM}_{5}$ & 1.450839 & 279.571 & 0.11 \\
\hline $\mathrm{TM}_{6}$ & 1.449356 & 344.685 & 0.02 \\
\hline $\mathrm{TM}_{7}$ & 1.448424 & 224.106 & 0.02 \\
\hline $\mathrm{TM}_{8}$ & 1.442591 & 59.822 & 0.13 \\
\hline $\mathrm{TM}_{9}$ & 1.440684 & 70.965 & \\
\hline & & & \\
\hline
\end{tabular}

what compensated from the loss difference between even and odd modes.

In conventional dual ARROWs, only the fundamental even and odd TE modes can propagate with negligible losses; all TM modes, even the fundamental ones, are not propagable due to the relatively small reflectivities from the Fabry-Pérot cavity. Therefore, an additional Fabry-Pérot cavity is inserted to sustain the fundamental even and odd TM modes while still keeping other modes leaky enough. As shown in Table I, for both polarizations, only the fundamental even and odd modes (modes 0 and 1 ) possess sufficiently low losses and high excitation efficiencies. Note that the excitation efficiencies are calculated with a Gaussian beam input. 


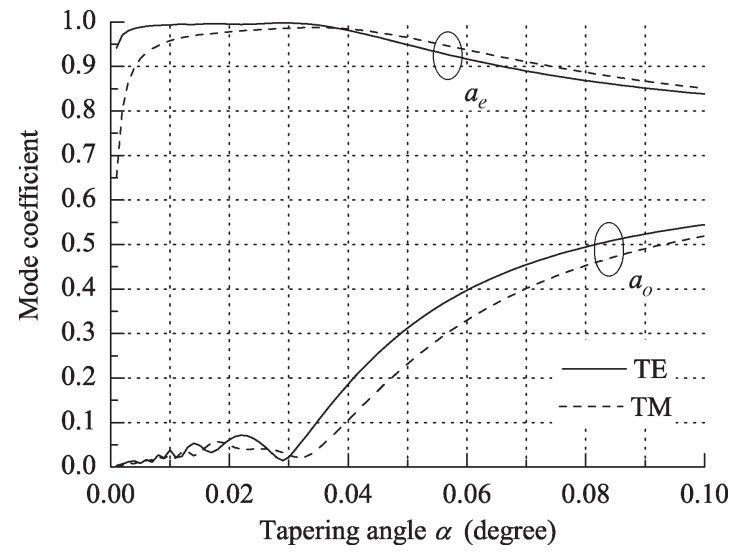

Fig. 8. Output mode coefficients $a_{e}$ and $a_{o}$ versus tapering angles for both polarizations, with the input condition of $\left[a_{e} a_{o}\right]=\left[\begin{array}{ll}1 & 0\end{array}\right]$.

The major design issue is the adiabatic condition of the tapered region. For analysis convenience, the tapered region is modeled as 2000 staircases with small and abrupt discontinuities. To determine the adiabatic invariance condition versus different tapering angles, we apply the eigenmode expansion method and launch only the fundamental even mode into the input end of the tapered region. If we express the field distribution as the superposition of the fundamental even and odd modes with corresponding expansion coefficients [16], then

$$
\psi(x, z)=a_{e} u_{e}(x) \exp \left(-\jmath \beta_{e} z\right)+a_{o} u_{o}(x) \exp \left(-\jmath \beta_{o} z\right)
$$

where $u(x)$ is the field distribution of the local normal mode, $\beta$ is the propagation constant, and $a_{e}$ and $a_{o}$ are the expansion coefficients of the fundamental even and odd modes, respectively. Then, the input condition can be written as $\left[\begin{array}{ll}a_{e} & a_{o}\end{array}\right]=\left[\begin{array}{ll}1 & 0\end{array}\right]$. The output mode coefficients for TE and TM polarizations are shown in Fig. 8. Note that the results are derived from the fact that the air-cladding layer is tapered from the input end with $d_{l_{1}}=1.91$ to $0 \mu \mathrm{m}$. As depicted in Fig. 8, with the tapering angles below $0.035^{\circ}$ for the TE polarization and $0.04^{\circ}$ for that of TM, there will be no significant mode conversion occurring (i.e., $a_{o}<0.1$ ); thus, we can arbitrarily choose $\alpha=0.025^{\circ}$ in this design example.

Afterward, the air-cladding layer thickness to which we taper from the input end is adjusted for minimizing the device length. Solving (7) and (9), we construct the solution diagram shown in Fig. 9. It can be found that with tapering the air-cladding layer to $0.153 \mu \mathrm{m}$, the optimum device length is $8.67 \mathrm{~mm}$, where the tuned-region length is $615 \mu \mathrm{m}$. Under this condition, the fundamental even and odd modes for the TE polarization are out of phase at the output end $(m=5)$; that is, the output power for the TE polarization mainly resides at core 2 , while that for TM at core $1(l=6)$. Fig. 10 illustrates how the effective indices evolve along the device for both polarizations, respectively. The area between the two lines multiplying $k_{0}$ is just the cumulated phase difference between the even and odd modes and is $5 \pi$ for TE-polarized and $6 \pi$ for TM-polarized waves, respectively. Fig. 11 shows the mode coefficients versus air-cladding layer thickness with tapering angle $\alpha=0.025^{\circ}$. It can be seen that $a_{o}$

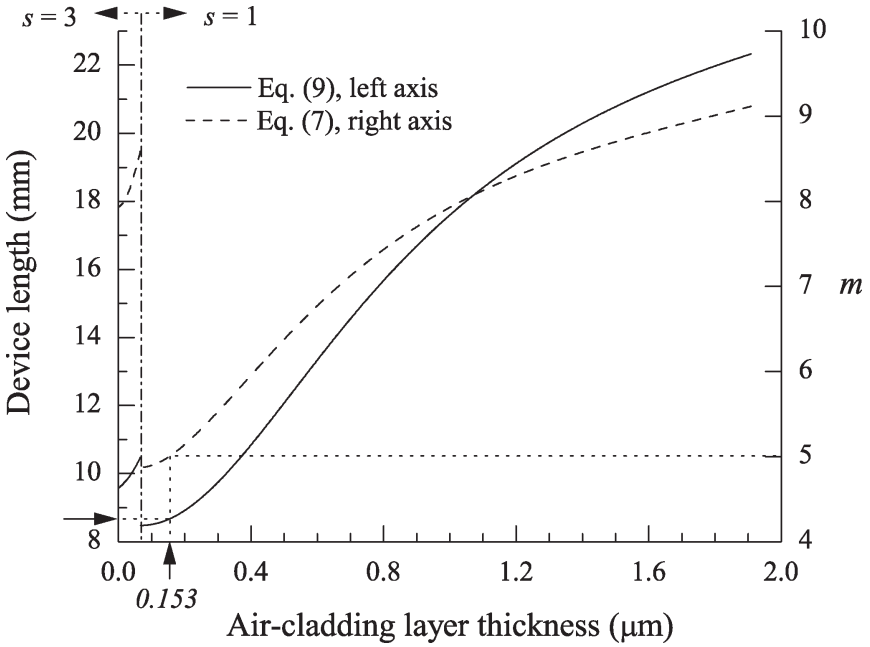

Fig. 9. Solution diagram for optimum design with tapering angle $\alpha=$ $0.025^{\circ}$.

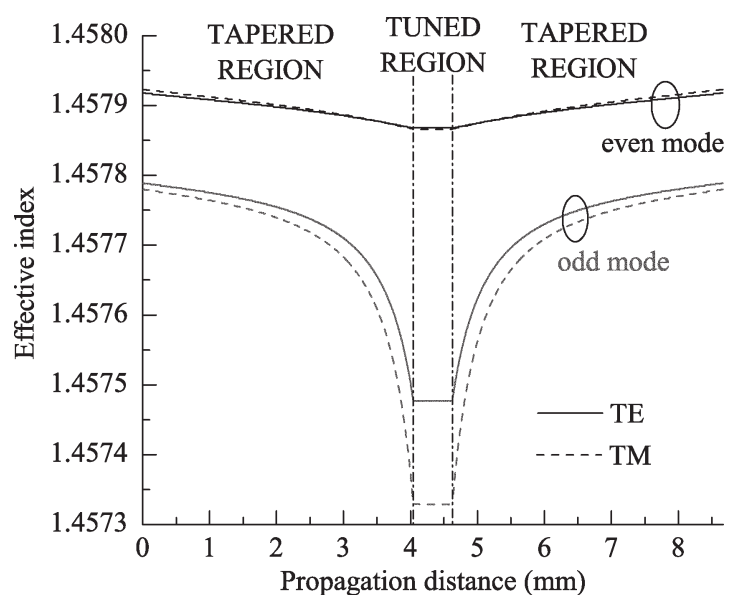

Fig. 10. Effective index evolutions along the device for TE and TM polarizations.

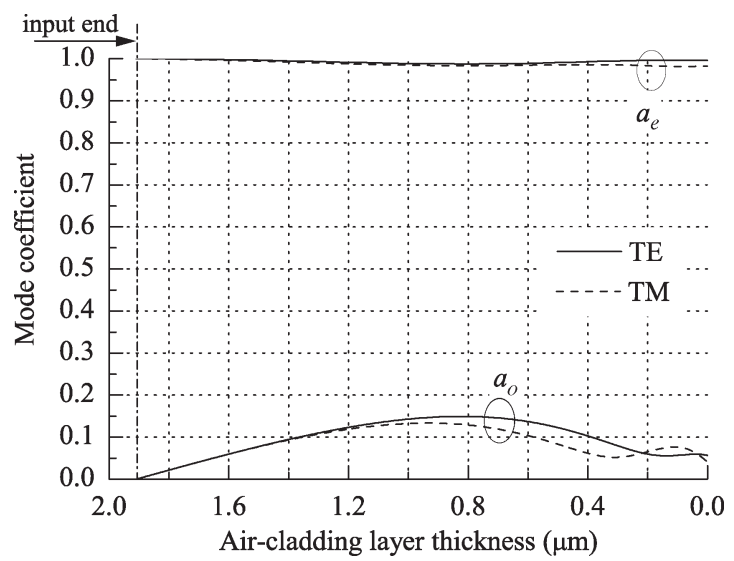

Fig. 11. Mode coefficients versus air-cladding layer thickness with tapering angle $\alpha=0.025^{\circ}$. The input condition is $\left[a_{e} a_{o}\right]=\left[\begin{array}{ll}1 & 0\end{array}\right]$.

is below 0.1 for either of both polarizations; that is, the adiabatic condition of the tapered region is preserved.

The FD-BPM simulation is applied to verify the design again. In either polarization, a unit-power wave composed of the superposition of even and odd modes is launched at core 1 . 

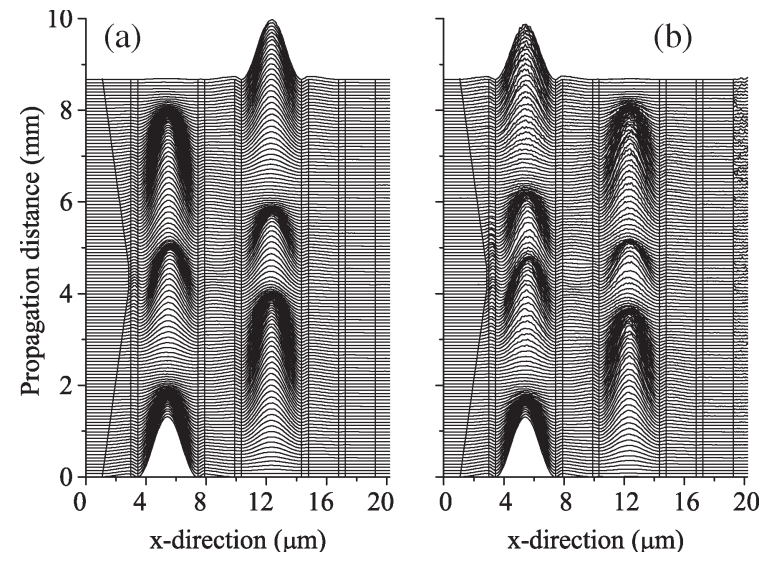

Fig. 12. Power evolution profiles along the propagation distance. (a) TE polarization. (b) TM polarization.

The power evolution profiles for the TE- and TM-polarized waves are shown in Fig. 12. As calculated from the results, the insertion losses for TE and TM polarizations are around 0.04 and $0.37 \mathrm{~dB}$, respectively, and the crosstalks of TE and TM waves are both approximately $-20 \mathrm{~dB}$. These somewhat high crosstalks are mainly attributed to the inexactly adiabatic condition of the tapered regions.

\section{Polarization Beam Splitter Based on ASYMMETRIC ARROW}

Although the idea of employing an adiabatically tapered ARROW structure can successfully shorten the device length of polarization beam splitters, the relatively high insertion loss difference between TE and TM polarizations, which is due to the inherent propagation loss difference of ARROW structures, is still an undesirable characteristic. If the polarization splitting can be accomplished during a single coupling process, not only the device length can be further minimized, but the insertion loss difference between polarizations will decrease as well. In this section, a polarization beam splitter based on an asymmetric dual ARROW waveguide is presented. With a suitable design, the two constituent ARROWs of this dual ARROW can be made optically identical for one polarization (TE) and nonidentical for the other (TM). That is, TE-polarized waves can couple between the two cores but TM waves cannot. By adjusting the device length as the coupling length of the TE polarization, the polarization splitting can be achieved.

We first consider a single ARROW structure (named ARROW 1) based on a material system of air $/ \mathrm{SiO}_{2} /$ glass mixture $/ \mathrm{SiO}_{2} / \mathrm{TiO}_{2} / \mathrm{SiO}_{2} / \mathrm{Si}$ with layer indices of $1.000 /$ $1.460 / 1.500 / 1.460 / 2.300 / 1.460 / 3.500$. The corresponding thicknesses of inner layers are 2.000/0.448/4.000/0.089/ $2.000 \mu \mathrm{m}$. As shown on the far right of Fig. $13\left(d_{l_{1}}=\right.$ $2.000 \mu \mathrm{m}$ ), the fundamental mode indices for both TE and TM polarizations are found to be approximately equal. As the air-cladding layer thickness $d_{l_{1}}$ decreases (to the left of Fig. 13), the difference between the effective indices of $\mathrm{TE}_{0}$ and $\mathrm{TM}_{0}$ becomes more significant. Then consider another single ARROW structure (ARROW 2) with the core index increased by 0.001 and the thicknesses of all layers adjusted

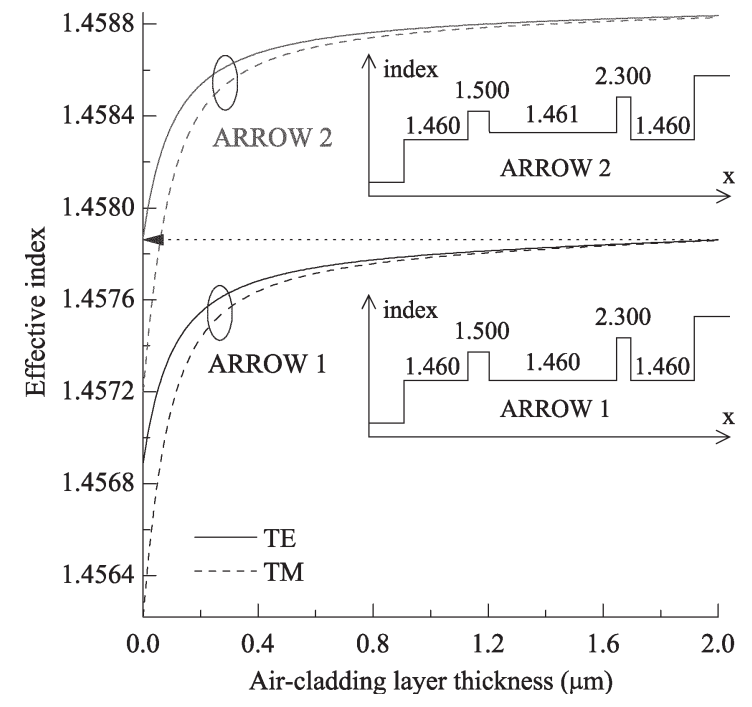

Fig. 13. Effective indices of $\mathrm{TE}_{0}$ and $\mathrm{TM}_{0}$ modes versus the air-cladding layer thicknesses of ARROW 1 and ARROW 2.

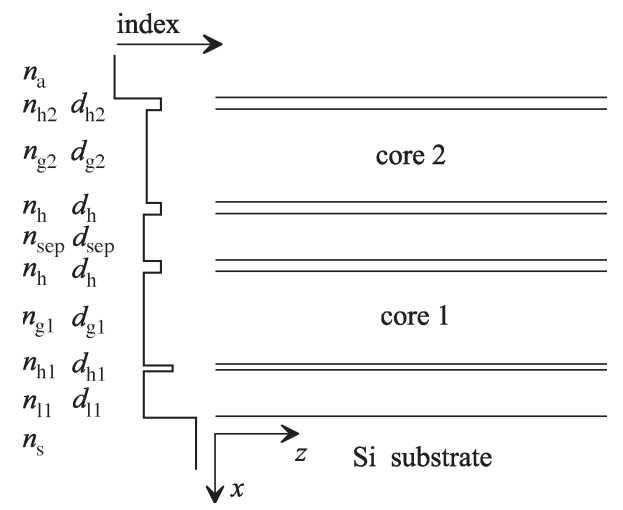

Fig. 14. Schematic view of the polarization beam splitter based on asymmetric ARROW.

correspondingly. When $d_{l_{1}}$ of ARROW 2 approaches zero and that of ARROW 1 remains at $2.000 \mu \mathrm{m}$, it can be seen that the $\mathrm{TE}_{0}$ mode indices of ARROW 1 and ARROW 2 are almost matched, whereas those of $\mathrm{TM}_{0}$ are separated with a large gap.

Based on this concept, we combine these two individual ARROWs to form a dual waveguide for polarization splitting, as illustrated in Fig. 14. The refractive index profile is 1.000/1.500/1.461/1.500/1.460/1.500/1.460/2.300/1.460/3.500, where the corresponding thicknesses of inner layers are $0.453 / 4.000 / 0.448 / 2.000 / 0.448 / 4.000 / 0.089 / 2.000 \mu \mathrm{m}$. The field distributions of the fundamental even and odd modes for TE and TM polarizations are plotted in Fig. 15(a) and (b), respectively. It is expected that the maximum coupling efficiency for TE polarization would be $C_{0, \mathrm{TE}} \approx 100 \%$, whereas that for TM would be $C_{0, \mathrm{TM}} \approx 0 \%$ [15]. When an optical beam containing both polarizations is launched into core 1 , for the TE polarization, the fundamental even and odd modes are both excited, whereas for the TM polarization, only the fundamental even mode will be highly excited. Therefore, choosing the device length $L$ as the beat length of the fundamental even and odd modes for the TE polarization, i.e., $L=\lambda /\left(2\left|N_{\mathrm{TE}, e}-N_{\mathrm{TE}, o}\right|\right)=1919 \mu \mathrm{m}$, the power of the TE-polarized wave will couple into core 2 , whereas that 

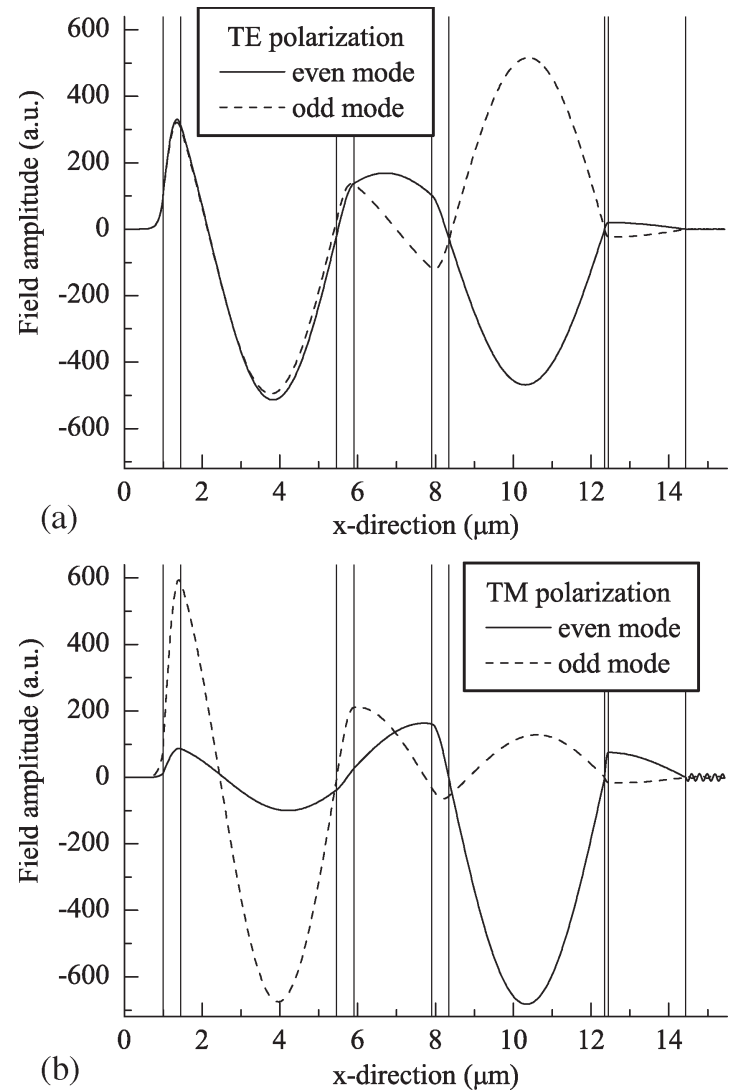

Fig. 15. Field distributions of the fundamental even and odd modes. (a) TE polarization. (b) TM polarization.
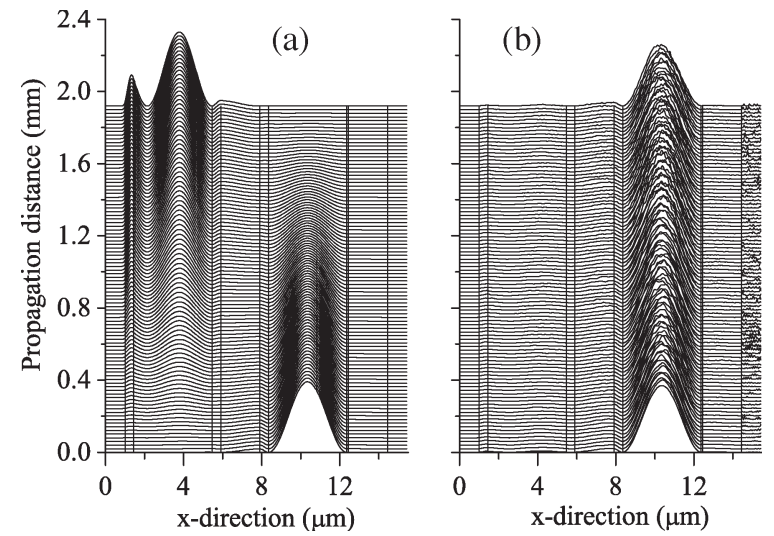

Fig. 16. Power evolution profiles. (a) TE polarization. (b) TM polarization.

of the TM-polarized wave will still remain at core 1 , and polarization splitting is obtained. Fig. 16(a) and (b) shows the BPM simulation results of the power evolution profiles for TEand TM-polarized waves, respectively.

It is understood that the variation of $n_{g_{2}}$ might be the dominant design concern. To investigate the allowed tolerance of the device on $n_{g_{2}}$, the crosstalks for TE and TM polarizations are used as a measure, as depicted in Fig. 17. For good polarization discrimination with crosstalks below $-20 \mathrm{~dB}$, it appears that the stringent tolerance of $\pm 1.5 \times 10^{-5}$ for $n_{g_{2}}$ is required. On the other hand, one feature of the device is its relatively short device length, and the length is short enough, so that the power

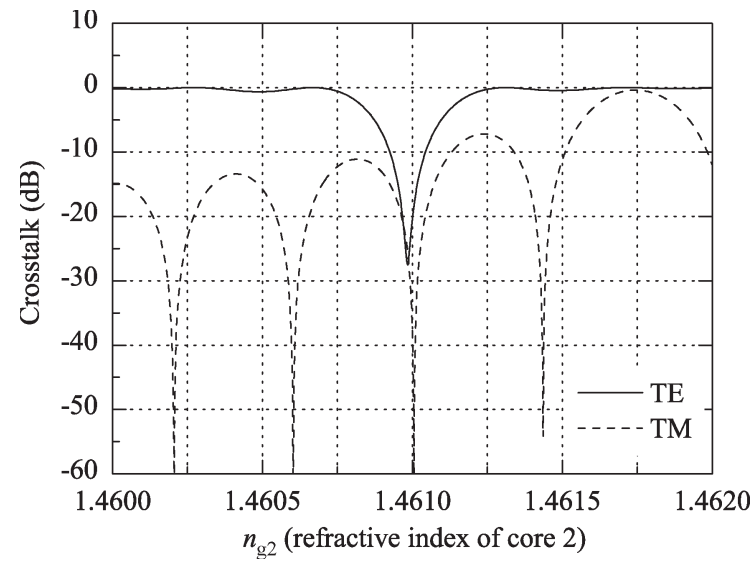

Fig. 17. Tolerance analysis of the device on $n_{g_{2}}$.

leakage of the TM-polarized wave is relatively small $(\approx 0.4 \mathrm{~dB})$ and close to that of the TE wave as well.

\section{CONCLUSION}

Three different configurations of ARROW polarization beam splitters are proposed. The operating schemes are of two types: one is creating a difference of the beat length in two polarization states (the first two cases), and the other is designing two waveguide channels optically identical for one polarization while nonidentical for the other (the third case).

Basically, the following characteristics of conventional ARROW and ARROW-B have been exploited in implementation of polarization splitting scheme: 1) the capability of ARROW-B structures in sustaining the power of TM-polarized waves, 2) the different reflectivities of TE- and TM-polarized waves confronting the Fabry-Pérot cavities, and 3) the dispersion relation between the fundamental mode index and the air-cladding layer thickness of an ARROW. Features 1 and 2 are utilized in the first case for polarization splitting. However, the drawbacks are its relatively lengthy dimension and large difference in insertion losses for two polarizations. In the second design, based on a dual ARROW configuration, we utilize feature 3 for polarization splitting by tapering the thickness of the air-cladding layer. The major requirement is that the tapering should be gradual enough to meet the adiabatic invariance condition. For the third type, also on the basis of a dual ARROW, we manipulate the upper waveguide and utilize feature 3 to achieve polarization splitting. However, the output field profile of one polarization is somewhat deviated. An approach by a tapered ARROW could be applied to resume the profile.

In summary, the lengths of the proposed devices range from about $26 \mathrm{~mm}$ to less than $2 \mathrm{~mm}$, and the crosstalks of both polarizations below $-20 \mathrm{~dB}$ are retained. Among the three polarization beam splitters, the concept of the first one is most straightforward, whereas its relatively long length and large insertion loss difference limit its usability. The second device can successfully reduce the length through a somewhat complicated design flow and a little extra effort to control the tapering. For the third case, not only the device length is shortest, but the losses are lowest as well. Although realizing 
the designs experimentally may raise some concerns over the tolerance, the FD-BPM has been applied for the verification of each design, and the simulation results have indicated that the presented devices are feasible.

\section{REFERENCES}

[1] O. Mikami, "LiNbO 3 coupled-waveguided TE/TM mode splitter," Appl. Phys. Lett., vol. 36, no. 7, pp. 491-493, Apr. 1980.

[2] H. Nakajima, T. Horimatsu, M. Seino, and I. Sasaki, "Crosstalk characteristics of $\mathrm{Ti}-\mathrm{LiNbO}_{3}$ intersecting waveguides and their application as TE/TM mode splitters," IEEE J. Quantum Electron., vol. QE-18, no. 4, pp. 771-776, Apr. 1982.

[3] Y. Shani, C. H. Henry, R. C. Kistler, and K. J. Orlowsky, "Fourport integrated optic polarization splitter," Appl. Opt., vol. 29, no. 3, pp. 337-339, Jan. 1990.

[4] I. Kiyat, A. Aydinli, and N. Dagli, "A compact silicon-on-insulator polarization splitter," IEEE Photon. Technol. Lett., vol. 17, no. 1, pp. 100-102, Jan. 2005.

[5] N. Goto and G. L. Yip, "A TE-TM mode splitter in $\mathrm{LiNbO}_{3}$ by proton exchange and Ti diffusion," J. Lightw. Technol., vol. 7, no. 10, pp. 15671574 , Oct. 1989.

[6] Y. Shani, C. H. Henry, R. C. Kistler, R. F. Kaazarinov, and K. J. Orlowsky, "Integrated optic adiabatic polarization splitter on silicon," Appl. Phys. Lett., vol. 56, no. 2, pp. 120-121, Jan. 1990.

[7] R. M. de Ridder, A. F. M. Sander, A. Driessen, and J. H. J. Fluitman, "Integrated optic adiabatic TE/TM mode splitter on silicon," J. Lightw. Technol., vol. 11, no. 11, pp. 1806-1811, Nov. 1993.

[8] S. M. Garner, V. Chuyanov, S.-S. Lee, A. Chen, W. H. Steier, and L. R. Dalton, "Vertically integrated waveguide polarization splitters using polymers," IEEE Photon. Technol. Lett., vol. 11, no. 7, pp. 842-844, Jul. 1999.

[9] E. Simova and I. Golub, "Polarization splitter/combiner in high index contrast Bragg reflector waveguides," Opt. Express, vol. 11, no. 25, pp. 3425-3430, Dec. 2003.

[10] T. Thyagarajan and S. Pilevar, "Resonant tunneling three-waveguide polarization splitter," J. Lightw. Technol., vol. 10, no. 10, pp. 1334-1337, Oct. 1992.

[11] U. Trutschel, F. Ouellette, V. Delisle, M. A. Duguay, G. Fogarty, and F. Lederer, "Polarization splitter based on antiresonant reflecting optical waveguides," J. Lightw. Technol., vol. 13, no. 2, pp. 239-243, Feb. 1995.

[12] M. A. Duguay, Y. Kokubun, T. L. Koch, and L. Pfeiffer, "Antiresonant reflecting optical waveguides in $\mathrm{SiO}_{2}-\mathrm{Si}$ multilayer structures," Appl. Phys. Lett., vol. 49, no. 1, pp. 13-15, Jul. 1986.

[13] J. Gehler, A. Bräuer, W. Karthe, and M. Jäger, "Antiresonant reflecting optical waveguides in strip configuration," Appl. Phys. Lett., vol. 64, no. 3, pp. 276-278, Jan. 1994.

[14] J. Gehler, A. Bräuer, and W. Karthe, "Remote coupling over $93 \mu \mathrm{m}$ using ARROW waveguides in strip configuration," Electron. Lett., vol. 30, no. 3 , pp. 218-220, Feb. 1994.

[15] Y.-H. Chen and Y.-T. Huang, "Coupling efficiency analysis and control of dual antiresonant reflecting optical waveguides," J. Lightw. Technol., vol. 14, no. 6, pp. 1507-1513, Jun. 1996.

[16] J.-J. Deng and Y.-T. Huang, "A novel hybrid coupler based on antiresonant reflecting optical waveguides," J. Lightw. Technol., vol. 16, no. 6, pp. 1062-1069, Jun. 1998

[17] T. Baba and Y. Kokubun, "New polarization-insensitive antiresonant reflecting optical waveguide (ARROW-B)," IEEE Photon. Technol. Lett., vol. 1, no. 8, pp. 232-234, Aug. 1989.

[18] M. Mann, U. Trutschel, C. Wachter, L. Leine, and F. Lederer, "Directional coupler based on antiresonant reflecting optical waveguides," Opt. Lett., vol. 16, no. 11, pp. 805-807, Jun. 1991.

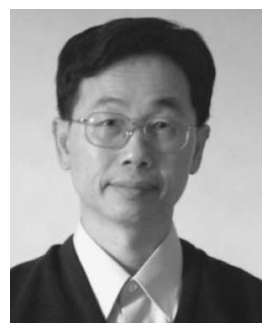

Yang-Tung Huang (M'90) was born in Taiwan, R.O.C., in 1955. He received the B.S. degree in electrophysics and the M.S. degree in electronics from the National Chiao Tung University, Hsinchu, Taiwan, in 1978 and 1982, respectively, and the Ph.D degree in electrical and computer engineering from the University of Arizona, Tuscon, in 1990.

$\mathrm{He}$ is a Professor with the Department of Electronics Engineering and the Institute of Electronics, National Chiao Tung University, and has been the Director of Nano Facility Center since 2003. He has been the Director of Institute of Electronics for three years and the Director of Semiconductor Research Center for two years. His current research interests include integrated optics, photonic crystal waveguides, biooptoelectronics, and optoelectronic switching networks.

Dr. Huang received the Outstanding Research Award from the National Science Council, Taiwan, R.O.C., in 1998

Chiou-Hung Jang received the B.S. degree in physics from the National Tsing Hua University, Hsinchu, Taiwan, R.O.C., in 1995, the M.S. degree in electronics from the National Chiao Tung University, Hsinchu, in 1997, and the $\mathrm{Ph} . \mathrm{D}$. degree in electrical and computer engineering from the University of Texas at Austin in 2002.

He is currently a Senior Staff Engineer with Intel Corporation, Hillsboro, $\mathrm{OR}$, working on lithography resolution enabling techniques.

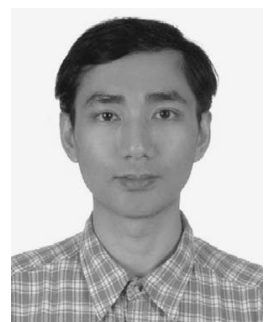

Shih-Hsin Hsu (S'99-M'06) received the B.S. degree in physics from the National Taiwan University, Taipei, Taiwan, R.O.C., in 1994 and the Ph.D. degree in electronics from the National Chiao Tung University, Hsinchu, Taiwan, in 2005.

$\mathrm{He}$ is a Postdoctoral Research Fellow with the Research Center for Applied Sciences, Academia Sinica, Taipei. His current research is focused on nanophotonics and optical characterizations.

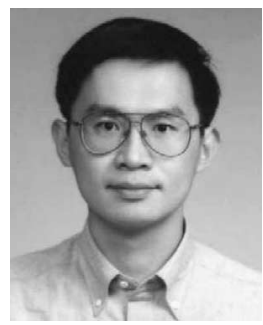

Jau-Jan Deng (S'92-M'00) received the B.S. degree in electronics engineering and the Ph.D. degree in electronics from the National Chiao Tung University, Hsinchu, Taiwan, R.O.C., in 1992 and 2000, respectively.

$\mathrm{He}$ is currently the R\&D Director with E-Pin Optical Industry Co., Taipei, Taiwan. 